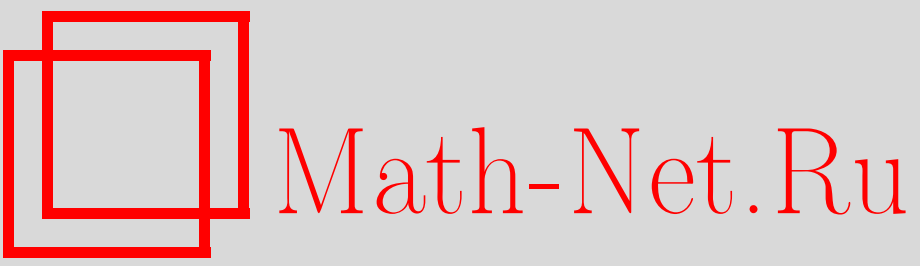

Л. П. Гинзбург, Обменно-корреляционный потенциал в методе функционала плотности, ТМФ, 2006, том 146, номер 2, 329-339

DOI: https://doi.org/10.4213/tmf2038

Использование Общероссийского математического портала Math-Net.Ru подразумевает, что вы прочитали и согласны с пользовательским соглашением

http://www.mathnet.ru/rus/agreement

Параметры загрузки:

IP: 3.85 .73 .92

26 апреля 2023 г., 18:36:51 
ТЕОРЕТИЧЕСКАЯ

И МАТЕМАТИЧЕСКАЯ

ФИЗИКА

Том 146, № 2

февраль, 2006

(C) 2006 г.

Л. П. Гинзбург*

\section{ОБМЕННО-КОРРЕЛЯЦИОННЫЙ ПОТЕНЦИАЛ В МЕТОДЕ ФУНКЦИОНАЛА ПЛОТНОСТИ}

\footnotetext{
Анализируется уравнение для высшего заполненного состояния в системе невзаимодействующих электронов в методе функционала плотности. Более строго, чем в известных на сегодняшний день работах, показано, что собственное значение уравнения определяет энергию ионизации. При этом существенно уточняется выражение для обменно-корреляционного потенциала.
}

Ключевые слова: многоэлектронная система, функционал плотности, обменнокорреляционный потенциал, верхнее заполненное состояние, одноэлектронное уравнение Шредингера.

\section{1. ВВЕДЕНИЕ}

В последнее время при изучении $N$-электронных систем $(N \geqslant 2)$ все бо́льшую популярность приобретает (сформулированный еще в 1964 г.) метод функционала плотности (см. работу [1] и ссылки в ней). По всей видимости, привлекательность метода состоит в том, что систему оказывается возможным описать в терминах воображаемой системы невзаимодействующих электронов, подчиненных уравнениям

$$
H(D F) \varphi_{i}(\mathbf{x})=e_{i} \varphi_{i}(\mathbf{x}),
$$

где $\mathbf{x}=(\mathbf{r}, \sigma), \sigma-$ спин электрона и

$$
H(D F)=-\frac{1}{2} \nabla^{2}+v(\mathbf{r})+\int d \mathbf{r}^{\prime} \frac{\rho\left(\mathbf{r}^{\prime}\right)}{\left|\mathbf{r}-\mathbf{r}^{\prime}\right|}+v_{\mathrm{xc}}(\mathbf{r}) .
$$

В соотношении (2) (где, как и везде в дальнейшем, использованы атомные единицы: $\left.\hbar=m=e_{0}^{2} /\left(4 \pi \varepsilon_{0}\right)=1\right) \quad v(\mathbf{r})$ - заданный внешний потенциал, $\rho(\mathbf{r})-$ электронная плотность, связанная с диагональным элементом матрицы плотности соотношением

$$
\rho(\mathbf{r})=N \gamma(\mathbf{r}, \mathbf{r})=N \sum_{\sigma} \gamma(\mathbf{x}, \mathbf{x}),
$$

* Московский технический университет связи и информатики, Москва, Россия. E-mail: italtrade@mtu-net.ru 
и $v_{\text {хс }}$ - так называемый обменно-корреляционный потенциал, представляющий собой вариационную производную

$$
v_{\mathrm{xc}}(\mathbf{r})=\frac{\delta E_{\mathrm{xc}}[\rho(\mathbf{r})]}{\delta \rho(\mathbf{r})},
$$

где $E_{\mathrm{xc}}[\rho]$ - фукционал от $\rho(\mathbf{r})$, который должен учитывать все обменно-корреляционные вклады в энергию и который в каждом конкретном случае подлежит определению. Очевидно, успех метода зависит от степени точности определения $E_{\mathrm{xc}}[\rho]$ и соответственно $v_{\text {хс }}(\mathbf{r})$.

В общем случае приписывать какой-либо смысл собственным состояниям уравнения (1) затруднительно [1]. Важно, однако, то, что они приводят к правильному выражению для $\rho(\mathbf{r})$ :

$$
\rho(\mathbf{r})=\sum_{\sigma} \sum_{i} \varphi_{i}^{*}(\mathbf{x}) \varphi_{i}(\mathbf{x})
$$

Последнее обстоятельство заставляет более внимательно посмотреть на то уравнение из набора (1), которое относится к самому высокому заполненному состоянию:

$$
H(D F) \varphi_{N}=e_{N} \varphi_{N}
$$

Совершенно очевидно, что при больших $r$, когда электрон удален от остальной $(N-1)$-электронной системы (которую мы будем называть остатком), с экспоненциальной точностью должно выполняться соотношение [2]

$$
\sum_{i} \varphi_{i}^{*}(\mathbf{x}) \varphi_{i}(\mathbf{x})=\varphi_{N}^{*}(\mathbf{x}) \varphi_{N}(\mathbf{x})
$$

Так как согласно выражению (5) в этом случае функция $\varphi_{N}(\mathbf{x})$ должна определять реальное распределение электронной плотности, то возникает сильное подозрение, что величина $e_{N}$ в уравнении (6) должна определять энергию ионизации:

$$
e_{N}=E_{N}-E_{N-1}^{0}=-I_{0}
$$

В соотношении (8) $E_{N}$ - энергия основного состояния системы, а $E_{N-1}^{0}-$ энергия основного состояния остатка. Обосновать соотношение (8) возможно на пути независимого вывода уравнения Шредингера для $\varphi_{N}(\mathbf{x})$ и сравнения его с $(6),(2)$. При этом (что не менее важно) должно получиться явное выражение для $v_{\text {хс }}(\mathbf{r})$. Наиболее последовательно сформулированная задача решалась в работе [2]. На базе (записанного в представлении вторичного квантования) уравнения для основного состояния системы

$$
H(N) \Psi(N)=E_{N} \Psi(N)
$$

и разложения

$$
\Psi(N)=\sum_{s} f_{s}(\mathbf{x})|N-1, s\rangle
$$


где $|N-1, s\rangle$ - волновая функция $s$-состояния остатка, в работе [2] в основу анализа положено уравнение

$$
\begin{gathered}
\left(-\frac{1}{2} \nabla^{2}+v(\mathbf{r})+\left\langle N-1, s\left|\sum_{j \geqslant 2} \frac{1}{\left|\mathbf{r}-\mathbf{r}_{j}\right|}\right| N-1, s\right\rangle\right) f_{s}(\mathbf{x})+ \\
+\sum_{s^{\prime} \neq s}\left\langle N-1, s\left|\sum_{j \geqslant 2} \frac{1}{\left|\mathbf{r}-\mathbf{r}_{j}\right|}\right| N-1, s^{\prime}\right\rangle f_{s^{\prime}}(\mathbf{x})=\varepsilon_{s} f_{s}(\mathbf{x}), \\
\varepsilon_{s}=E_{N}-E(N-1, s)
\end{gathered}
$$

$E(N-1, s)$ - энергия $s$-состояния остатка. Из краткого изложения основных положений работы [2] (см. раздел 2) видно, что соотношение (8), а также выражения

$$
\begin{gathered}
v_{\mathrm{xc}}(\mathbf{r})=\left\langle N-1,0\left|\sum_{j \geqslant 2} \frac{1}{\left|\mathbf{r}-\mathbf{r}_{j}\right|}\right| N-1,0\right\rangle- \\
-\int d \mathbf{r}^{\prime} \frac{\rho\left(\mathbf{r}^{\prime}\right)}{\left|\mathbf{r}-\mathbf{r}^{\prime}\right|}-\frac{1}{r^{4}} \sum_{s>0} \frac{\left|D_{s 0}\right|^{2}}{\omega_{s}},
\end{gathered}
$$

где $s=0$ соответствует основному состоянию, и

$$
\begin{gathered}
D_{s s^{\prime}}=\left\langle N-1, s\left|\sum_{j \geqslant 2} r_{j} \cos \theta_{j}\right| N-1, s^{\prime}\right\rangle, \\
\omega_{s}=E(N-1, s)-E_{N-1}^{0}
\end{gathered}
$$

получены в работе [2] путем использования ограничений более жестких, чем (7), а именно разложения (17) (см. ниже), а также отбрасывания в гамильтониане величин порядка $O\left(1 / r^{n}\right)$, где $n>4$. По этой причине в разделе 3 настоящей работы предлагается альтернативное обоснование соотношения (8) путем вывода уравнения Шредингера для функции

$$
\phi(\mathbf{r})=\left(\frac{1}{N} \rho(\mathbf{r})\right)^{1 / 2} .
$$

При этом никакие другие ограничения, кроме (7), не используются. Раздел 4 посвящен сравнению полученного таким путем выражения для $v_{\text {хс }}(\mathbf{r})$ и формулы (13). Обнаруживаются определенные расхождения, природа которых обсуждается в разделе 5 .

\section{2. ВЫВОД ФОРМУЛЫ ДЛЯ ОБМЕННО-КОРРЕЛЯЦИОННОГО ПОТЕНЦИАЛА}

В данном разделе рассматриваются основные положения работы [2]. В ней обращается внимание на то, что если в условиях $r>r_{j}$ воспользоваться соотношением

$$
\frac{1}{\left|\mathbf{r}-\mathbf{r}_{j}\right|}=\frac{1}{r}+\frac{r_{j}}{r^{2}} \cos \theta_{j}
$$


где $\theta_{j}-$ угол между векторами $\mathbf{r}$ и $\mathbf{r}_{j}$, то монопольный член вклада в сумму в левой части (11) не дает. Поэтому можно написать

$$
\left(-\frac{1}{2} \nabla^{2}+v(\mathbf{r})+\left\langle N-1, s\left|\sum_{j \geqslant 2} \frac{1}{\left|\mathbf{r}-\mathbf{r}_{j}\right|}\right| N-1, s\right\rangle-\varepsilon_{s}\right) f_{s}(\mathbf{x})=-\frac{1}{r^{2}} \sum_{s^{\prime} \neq s} D_{s s^{\prime}} f_{s^{\prime}}(\mathbf{x}) .
$$

Соответственно для основного состояния

$$
\left(-\frac{1}{2} \nabla^{2}+v(\mathbf{r})+\left\langle N-1,0\left|\sum_{j \geqslant 2} \frac{1}{\left|\mathbf{r}-\mathbf{r}_{j}\right|}\right| N-1,0\right\rangle-\varepsilon_{0}\right) f_{0}(\mathbf{x})=-\frac{1}{r^{2}} \sum_{s>0} D_{0 s} f_{s}(\mathbf{x}) .
$$

Далее в работе [2] констатируется, что согласно выражению (19) асимптотическое (при больших $r$ ) поведение функции $f_{0}(\mathbf{x})$ должно определяться соотношением $e^{-\left(2 \varepsilon_{0}\right)^{1 / 2} r}$. Так как в сумму в правой части выражения (18) входит слагаемое, содержащее $f_{0}(\mathbf{x})$, то отсюда делается вывод, что все функции $f_{s}(\mathbf{x})$ при $s>0$ должны иметь такую же асимптотику, что и $f_{0}(\mathbf{x})$. Этому требованию можно удовлетворить, если считать, что в пределе больших $r$ выполняется соотношение

$$
\left(-\frac{1}{2} \nabla^{2}-\varepsilon_{s}\right) f_{s}(\mathbf{x}) \sim\left(\varepsilon_{0}-\varepsilon_{s}\right) f_{s}(\mathbf{x}) .
$$

Полагая, что в области рассматриваемых значений $r$ вторым и третьим членами в левой части выражения (18) можно пренебречь по сравнению с $\varepsilon_{0}-\varepsilon_{s}$, мы из соотношений $(18),(20)$ получим

$$
\left(\varepsilon_{s}-\varepsilon_{0}\right) f_{s}(\mathbf{x}) \sim \frac{1}{r^{2}} \sum_{s^{\prime} \neq s} D_{s s^{\prime}} f_{s^{\prime}}(\mathbf{x}) .
$$

Отсюда видно, что каждое слагаемое в правой части при $s^{\prime}>0$ будет содержать фактор $\sim 1 / r^{2}$. Поэтому при $s>0$

$$
f_{s}(\mathbf{x}) \sim-\frac{1}{r^{2}} \frac{D_{s 0}}{\omega_{s}} f_{0}(\mathbf{x}) .
$$

Подставив соотношение (22) в выражение (19), получим уравнение

$$
\left(-\frac{1}{2} \nabla^{2}+v(\mathbf{r})+\left\langle N-1,0\left|\sum_{j \geqslant 2} \frac{1}{\left|\mathbf{r}-\mathbf{r}_{j}\right|}\right| N-1,0\right\rangle-\frac{1}{r^{4}} \sum_{s>0} \frac{\left|D_{s 0}\right|^{2}}{\omega_{s}}\right) f_{0}(\mathbf{x})=\varepsilon_{0} f_{0}(\mathbf{x}) .
$$

Согласно формулам (10) и (22), для зависящей от спина диагональной матрицы плотности можно написать

$$
\gamma(x, x)=\sum_{s} f_{s}^{*}(x) f_{s}(x) \cong f_{0}^{*}(\mathbf{x}) f_{0}(\mathbf{x})\left(1+\frac{1}{r^{4}} \sum_{s>0} \frac{\left|D_{s 0}\right|^{2}}{\omega_{s}^{2}}\right) .
$$

Сравнивания выражение (24) с (5) и (7), можно сделать вывод, что

$$
\left(\frac{1}{N}\right)^{1 / 2} \varphi_{N}(\mathbf{x})=f_{0}(\mathbf{x})\left(1+0\left(\frac{1}{r^{4}}\right)\right) \text {. }
$$

Подставив в уравнение $(23)$ вместо функций $f_{0}(\mathbf{x})$ функцию $\varphi_{N}(\mathbf{x})$ и сравнив полученное уравнение с (6) и (2), а также с (12), приходим к соотношениям (8) и (13). 


\section{3. АЛЬТЕРНАТИВНЫЙ ВЫВОД УРАВНЕНИЯ ДЛЯ ЭНЕРГИИ ИОНИЗАЦИИ}

В работах [3], [4] выведено точное одноэлектронное уравнение

$$
\left(H_{1}+E_{N-1}(\mathbf{r})\right) \phi(r)=E_{N} \phi(\mathbf{r}),
$$

в котором $\phi(\mathbf{r})$ определяется формулой (16).

Уравнение (26) получено посредством применения к уравнению (9) факторизации

$$
\Psi(N)=\phi(\mathbf{r}) \Phi\left(\mathbf{r}, \sigma ; \mathbf{X}_{N-1}\right) \equiv \phi(\mathbf{r}) \Phi(N-1) .
$$

В результате в уравнении (26)

$$
\begin{gathered}
H_{1}=-\frac{1}{2} \nabla^{2}+\frac{1}{2} \sum_{\sigma} \int d \mathbf{X}_{N-1} \nabla \Phi^{*}(N-1) \nabla \Phi(N-1)+v(\mathbf{r})+\langle U(\mathbf{r})\rangle, \\
\langle U(\mathbf{r})\rangle=\sum_{\sigma} \int d \mathbf{X}_{N-1} \Phi^{*}(N-1) \sum_{j \geqslant 2} \frac{1}{\left|\mathbf{r}-\mathbf{r}_{j}\right|} \Phi(N-1), \\
E_{N-1}(r)=\sum_{\sigma} \int d \mathbf{X}_{N-1} \Phi^{*}(N-1) H(N-1) \Phi(N-1),
\end{gathered}
$$

где $H(N-1)$ - гамильтониан остатка и $\mathbf{X}_{N-1}=\left(\mathbf{x}_{2}, \ldots, \mathbf{x}_{N}\right)$, так что

$$
\int d \mathbf{X}_{N-1}=\int d \mathbf{x}_{2} \ldots \int d \mathbf{x}_{N}, \quad \int d \mathbf{x}_{j}=\sum_{\sigma} \int d \mathbf{r}_{j} .
$$

Введем обозначение

$$
\sum_{\sigma} \sum_{s>0}|\langle N-1, s \mid \Phi(N-1)\rangle|^{2}=\theta(\mathbf{r})
$$

Учитывая, что [3], [4]

$$
\sum_{\sigma}\langle\Phi(N-1) \mid \Phi(N-1)\rangle \equiv \sum_{\sigma} \int d X_{N-1} \Phi^{*}(N-1) \Phi(N-1)=1,
$$

будем иметь

$$
\sum_{\sigma}|\langle N-1,0 \mid \Phi(N-1)\rangle|^{2}=1-\theta(\mathbf{r})
$$

Принимая во внимание соотношение

$$
H(N-1)|N-1, s\rangle=|N-1, s\rangle E(N-1, s)
$$

и воспользовавшись разложением

$$
\Phi(N-1)=\sum_{s}\langle N-1, s \mid \Phi(N-1)\rangle|N-1, s\rangle,
$$


из выражений (30), (34) получим

$$
\begin{aligned}
E_{N-1}(\mathbf{r})= & \sum_{\sigma} \sum_{s} E(N-1, s)|\langle\Phi(N-1) \mid N-1, s\rangle|^{2}= \\
= & E_{N-1}^{0} \sum_{\sigma}|\langle\Phi(N-1) \mid N-1,0\rangle|^{2}+ \\
& \quad+\sum_{\sigma} \sum_{s>0} E(N-1, s)|\langle\Phi(N-1) \mid N-1, s\rangle|^{2}= \\
= & E_{N-1}^{0}+\left(E_{N-1}^{*}-E_{N-1}^{0}\right) \theta(\mathbf{r}),
\end{aligned}
$$

где

$$
E_{N-1}^{*}=\frac{1}{\theta(\mathbf{r})} \sum_{\sigma} \sum_{s>0} E(N-1, s)|\langle\Phi(N-1) \mid N-1, s\rangle|^{2} .
$$

Поэтому, если принять во внимание равенство [4]

$$
\langle U(\mathbf{r})\rangle=\int d \mathbf{r}^{\prime} \frac{\rho\left(\mathbf{r}^{\prime}\right)}{\left|\mathbf{r}-\mathbf{r}^{\prime}\right|}+\int d \mathbf{r}^{\prime} \frac{\rho_{\mathrm{xc}}\left(\mathbf{r}, \mathbf{r}^{\prime}\right)}{\left|\mathbf{r}-\mathbf{r}^{\prime}\right|},
$$

где $\rho_{\mathrm{xc}}\left(\mathbf{r}, \mathbf{r}^{\prime}\right)$ - функция распределения ферми-кулоновской дырки [1], [5], то, подставив выражения (37), (39) в уравнение (26), мы получим уравнение

$$
\begin{aligned}
\left\{-\frac{1}{2} \nabla^{2}\right. & +\frac{1}{2} \sum_{\sigma} \int d \mathbf{X}_{N-1} \nabla \Phi^{*}(N-1) \nabla \Phi(N-1)+v(\mathbf{r})+\int d \mathbf{r}^{\prime} \frac{\rho\left(\mathbf{r}^{\prime}\right)}{\left|\mathbf{r}-\mathbf{r}^{\prime}\right|}+ \\
& \left.+\int d \mathbf{r}^{\prime} \frac{\rho_{\mathrm{xc}}\left(\mathbf{r}, \mathbf{r}^{\prime}\right)}{\left|\mathbf{r}-\mathbf{r}^{\prime}\right|}+\left(E_{N-1}^{*}-E_{N-1}^{0}\right) \theta(\mathbf{r})\right\} \phi(\mathbf{r})=\left(E_{N}-E_{N-1}^{0}\right) \phi(\mathbf{r}) .
\end{aligned}
$$

Согласно соотношениям (3), (5), (7), (16) в пренебрежении спин-орбитальным взаимодействием

$$
\phi^{*}(\mathbf{r}) \phi(\mathbf{r})=\sum_{\sigma} \varphi_{N}^{*}(\mathbf{x}) \varphi_{N}(\mathbf{x})=\varphi_{N}^{*}(\mathbf{r}) \varphi_{N}(\mathbf{r}) .
$$

Поэтому, сравнивая уравнение (40) с (6) и (2), убеждаемся в справедливости соотношения

$v_{\mathrm{xc}}(\mathbf{r})=\frac{1}{2} \sum_{\sigma} \int d \mathbf{X}_{N-1} \nabla \Phi^{*}(N-1) \nabla \Phi(N-1)+\int d \mathbf{r}^{\prime} \frac{\rho\left(\mathbf{r}^{\prime}\right)}{\left|\mathbf{r}-\mathbf{r}^{\prime}\right|}+\theta(\mathbf{r})\left(E_{N-1}^{*}-E_{N-1}^{0}\right)$.

\section{4. СРАВНЕНИЕ ОБМЕННО-КОРРЕЛЯЦИОННЫХ ПОТЕНЦИАЛОВ}

Как отмечалось, при выводе соотношений (40), (42) никаких дополнительных ограничений, кроме (7), не накладывалось (в отличие от работы [2]). В этом смысле соотношение (42) является более точным, чем (13). Поэтому представляется важным получить тот вид формулы (42), который она приобретает при использовании ограничений, рассматриваемых в работе [2] (основные из них - отбрасывание в гамильтониане всех величин, которые малы как $O\left(1 / r^{n}\right)$, где $n>4$, и использование (17) (см. раздел 2)). 
Прежде всего учтем, что согласно соотношениям (3), (24)

$$
\frac{1}{N} \rho(\mathbf{r})=\sum_{\sigma} f_{0}^{*}(\mathbf{x}) f_{0}(\mathbf{x})\left(1+\frac{1}{r^{4}} \sum_{s>0} \frac{\left|D_{s 0}\right|^{2}}{\omega_{s}^{2}}\right) .
$$

С другой стороны, на основании выражений (10), (27)

$$
f_{s}(\mathbf{x})=\phi(\mathbf{r})\langle N-1, s \mid \Phi(N-1)\rangle .
$$

Поэтому из формулы (43) следует, что

$$
\frac{1}{N} \rho(\mathbf{r})=\phi^{*}(\mathbf{r}) \phi(\mathbf{r}) \sum_{\sigma}|\langle N-1,0 \mid \Phi(N-1)\rangle|^{2}\left(1+\frac{1}{r^{4}} \sum_{s>0} \frac{\left|D_{s 0}\right|^{2}}{\omega_{s}^{2}}\right) .
$$

Приняв во внимание выражение (16), получим

$$
\sum_{\sigma}|\langle N-1,0 \mid \Phi(N-1)\rangle|^{2}\left(1+\frac{1}{r^{4}} \sum_{s>0} \frac{\left|D_{s 0}\right|^{2}}{\omega_{s}^{2}}\right)=1 .
$$

Отсюда, согласно (34),

$$
\theta(\mathbf{r})=\frac{1}{r^{4}} \sum_{s>0} \frac{\left|D_{s 0}\right|^{2}}{\omega_{s}^{2}}\left(1+\frac{1}{r^{4}} \sum_{s>0} \frac{\left|D_{s 0}\right|^{2}}{\omega_{s}^{2}}\right)^{-1}=\frac{1}{r^{4}} \sum_{s>0} \frac{\left|D_{s 0}\right|^{2}}{\omega_{s}^{2}},
$$

где отброшены величины $\sim 1 / r^{8}$. Теперь учтем, что из формул (29), (39) следует, что

$$
\int d \mathbf{r}^{\prime} \frac{\rho_{\mathrm{xc}}\left(\mathbf{r}, \mathbf{r}^{\prime}\right)}{\left|\mathbf{r}-\mathbf{r}^{\prime}\right|}=\sum_{\sigma} \int d \mathbf{X}_{N-1} \Phi^{*}(N-1) \sum_{j \geqslant 2} \frac{1}{\left|\mathbf{r}-\mathbf{r}_{j}\right|} \Phi(N-1)-\int d \mathbf{r}^{\prime} \frac{\rho\left(\mathbf{r}^{\prime}\right)}{\left|\mathbf{r}-\mathbf{r}^{\prime}\right|}
$$

Используя соотношения (34), (36), (47), можно записать

$$
\sum_{\sigma} \int d \mathbf{X}_{N-1} \Phi^{*}(N-1) \sum_{j \geqslant 2} \frac{1}{\left|\mathbf{r}-\mathbf{r}^{\prime}\right|} \Phi(N-1)=\left\langle N-1,0\left|\sum_{j \geqslant 2} \frac{1}{\left|\mathbf{r}-\mathbf{r}_{j}\right|}\right| N-1,0\right\rangle-L(\mathbf{r}),
$$

где

$$
\begin{aligned}
L(\mathbf{r})=\frac{1}{r^{4}} & \sum_{s>0} \frac{\left|D_{s 0}\right|^{2}}{\omega_{s}^{2}}\left\langle N-1,0\left|\sum_{j \geqslant 2} \frac{1}{\left|\mathbf{r}-\mathbf{r}_{j}\right|}\right| N-1,0\right\rangle- \\
& -\sum_{\sigma} \sum_{s>0} \sum_{s^{\prime}>0}\langle\Phi(N-1) \mid N-1, s\rangle \times \\
& \times\left\langle N-1, s\left|\sum_{j \geqslant 2} \frac{1}{\mathbf{r}-\mathbf{r}_{j}}\right| N-1, s^{\prime}\right\rangle\left\langle N-1, s^{\prime} \mid \Phi(N-1)\right\rangle .
\end{aligned}
$$

Для оценки $L(\mathbf{r})$ используем равенство (17). Приняв во внимание соотношения $(32),(47)$, легко убедиться в том, что монопольный член не дает вклада в $L(r)$. Поэтому, согласно (14),

$$
L(\mathbf{r})=\frac{D_{00}}{r^{6}} \sum_{s>0} \frac{\left|D_{s 0}\right|^{2}}{\omega_{s}^{2}}-\frac{1}{r^{2}} \sum_{\sigma} \sum_{s>0} \sum_{s^{\prime}>0}\langle\Phi(N-1) \mid N-1, s\rangle D_{s s^{\prime}}\left\langle N-1, s^{\prime} \mid \Phi(N-1)\right\rangle .
$$


На основании формул (16), (22), (43), (44)

$$
\begin{aligned}
& \sum_{\sigma} \sum_{s>0} \sum_{s^{\prime}>0}\langle\Phi(N-1) \mid N-1, s\rangle D_{s s^{\prime}}\left\langle N-1, s^{\prime} \mid \Phi(N-1)\right\rangle= \\
& \quad=\frac{N}{\rho(\mathbf{r})} \frac{1}{r^{4}} \sum_{\sigma} f_{0}^{*}(\mathbf{x}) f_{0}(\mathbf{x}) \sum_{s>0} \sum_{s^{\prime}>0} \frac{D_{0 s} D_{s s^{\prime}} D_{s^{\prime} 0}}{\omega_{s} \omega_{s^{\prime}}} \approx \frac{1}{r^{4}} \sum_{s>0} \sum_{s^{\prime}>0} \frac{D_{0 s} D_{s s^{\prime}} D_{s^{\prime} 0}}{\omega_{s} \omega_{s^{\prime}}} .
\end{aligned}
$$

Из (51), (52) следует, что при больших $r$

$$
|L(r)|=O\left(\frac{1}{r^{6}}\right)
$$

Такие члены в схеме, предложенной в работе [2], отбрасываются. Таким образом, в рамках приближений работы [2] из соотношений (48), (49), (53) следует

$$
\int d \mathbf{r}^{\prime} \frac{\rho_{x c}\left(\mathbf{r}, \mathbf{r}^{\prime}\right)}{\left|\mathbf{r}-\mathbf{r}^{\prime}\right|}=\left\langle N-1,0\left|\sum_{j \geqslant 2} \frac{1}{\left|\mathbf{r}-\mathbf{r}_{j}\right|}\right| N-1,0\right\rangle-\int d \mathbf{r}^{\prime} \frac{\rho\left(\mathbf{r}^{\prime}\right)}{\left|\mathbf{r}-\mathbf{r}^{\prime}\right|}
$$

что совпадает с первыми двумя членами выражения (13). Однако в соотношении (42) присутствуют еще два члена. Согласно формуле (47), последний член в соотношении (42) имеет такую же асимптотику, что и последний член выражения (13), но у них разные знаки. Что же касается первого члена соотношения (42), то в схеме, предложенной в работе [2], он не может появиться ни в каком приближении. Действительно, разложим функцию $|\Phi(N-1)\rangle$ по плоским волнам:

$$
|\Phi(N-1)\rangle=\sum_{\mathbf{k}}\langle\mathbf{k} \mid \Phi(N-1)\rangle|\mathbf{k}\rangle
$$

где

$$
|\mathbf{k}\rangle=\left(\frac{1}{\Omega}\right)^{1 / 2} e^{i \mathbf{k r}}
$$

и $\Omega$ - объем всей системы. Испльзуя выражения (55), (56), а также равенство

$$
\nabla e^{i \mathbf{k r}}=i \mathbf{k} e^{i \mathbf{k r}},
$$

мы будем иметь

$$
\begin{aligned}
& \frac{1}{2} \sum_{\sigma} \int d X_{N-1} \nabla \Phi^{*}(N-1) \nabla \Phi(N-1)= \\
& \quad=\frac{1}{2} \sum_{\sigma} \int d \mathbf{X}_{N-1} \frac{1}{\Omega} \sum_{\mathbf{k}} \sum_{\mathbf{k}^{\prime}} \mathbf{k k}^{\prime}\langle\Phi(N-1) \mid \mathbf{k}\rangle\left\langle\mathbf{k}^{\prime} \mid \Phi(N-1)\right\rangle e^{i\left(\mathbf{k}^{\prime}-\mathbf{k}\right) \mathbf{r}}
\end{aligned}
$$

Интегрируя выражение (58) по объему $\Omega$ и принимая во внимание, что

$$
\frac{8 \pi^{3}}{\Omega} \delta\left(\mathbf{k}^{\prime}-\mathbf{k}\right)=\delta_{\mathbf{k k}^{\prime}}
$$


получим

$$
\begin{aligned}
\frac{1}{2} \int d \mathbf{r} \sum_{\sigma} \int d \mathbf{X}_{N-1} \nabla \Phi^{*}(N-1) \nabla \Phi(N-1) & = \\
=\frac{1}{2} \sum_{\sigma} \int d \mathbf{X}_{N-1} \sum_{k} k^{2}|\langle\Phi(N-1) \mid \mathbf{k}\rangle|^{2} & =\frac{1}{2}\left(k^{2}\right)_{\text {эФФ }}
\end{aligned}
$$

где принято во внимание равенство (33). Очевидно, что $\left(k^{2}\right)_{\text {эфф }} \sim 1 / r^{2}$. При больших $r \Omega \sim r^{3}$. Поэтому асимптотика первого члена в соотношении (42) может быть оценена, как $O\left(1 / r^{5}\right)$. Из анализа, приведенного в разделе 2 , следует, что каждое последующее приближение в используемой в работе [2] схеме приводит к появлению фактора $1 / r^{2}$. Например, следующее приближение в оценке $f_{s}(\mathbf{x}), s>0$, согласно соотношениям (21), (22) будет иметь вид

$$
f_{s}(x)=\left(-\frac{1}{r^{2}} \frac{D_{s 0}}{\omega_{s}}+\frac{1}{r^{4}} \sum_{s^{\prime}} \frac{D_{s s^{\prime}} D_{s^{\prime} 0}}{\omega_{s} \omega_{s^{\prime}}}\right) f_{0}(x) .
$$

В выражении (13) при этом появится поправка $\sim 1 / r^{6}$, т.е. член $\sim 1 / r^{5}$ в схеме, применяемой в работе [2], отсутствует.

\section{5. ПРИРОДА РАСХОЖДЕНИЙ}

На наш взгляд, расхождения между формулами (13) и (42) связаны с тем, что в работе [2] не вполне оправданно приравниваются функции $f_{0}(\mathbf{x})$ и $\varphi_{N}(\mathbf{x})$. Эти функции относятся к разным системам и имеют различный физический смысл. С помощью выражений $(9),(10),(12)$ и соотношения

$$
H(N)=H(1)+H(N-1),
$$

где

$$
H(1)=-\frac{1}{2} \nabla^{2}+v(\mathbf{r})+\sum_{j \geqslant 2} \frac{1}{\left|\mathbf{r}-\mathbf{r}_{j}\right|},
$$

несложно установить, что уравнение (11) есть уравнение (9), переписанное в представлении оператора $H(N-1)$. Отсюда вытекают два следствия. Во-первых, уравнение (11) и все получаемые из него соотношения (в частности, уравнение (23)) должны, как и $\Psi(N)$, относиться к системе взаимодействующих электронов. Вовторых, функция $f_{0}(\mathbf{x})$ представляет собой зависящую от $\mathbf{r}, \sigma$ амплитуду условной вероятности того, что если вся система находится в состоянии $\Psi(N)$, то остаток оказывается в состоянии $s=0$. Поэтому уравнение $(23)$ - это на самом деле не уравнение Шредингера для внешнего электрона. Это уравнение для остатка, в котором указанный электрон фигурирует как возмущающий фактор.

По нашему мнению первое из отмеченных следствий ответственно за то, что в схеме, используемой в работе [2], принципиально не может появиться член, подобный первому члену в соотношении (42). Действительно, в системе взаимодействующих электронов кинетическая энергия должна определяться равенством

$$
T=\left\langle\Psi(N)\left|-\frac{1}{2} \sum_{i} \nabla_{i}^{2}\right| \Psi(N)\right\rangle .
$$


В работе [5] показано, что в такой системе обменно-корреляционный функционал определяется соотношением

$$
E_{\mathrm{xc}}^{\prime}[\rho]=\frac{1}{2} \int d \mathbf{r} \int d \mathbf{r}^{\prime}\left[\rho(\mathbf{r}) \frac{\rho_{\mathrm{xc}}\left(\mathbf{r}, \mathbf{r}^{\prime}\right)}{\left|\mathbf{r}-\mathbf{r}^{\prime}\right|}\right] .
$$

В случае системы невзаимодействующих электронов, которая описывается уравнениями (1), (6), вместо равенства (64) мы будем иметь

$$
T_{s}[\rho]=\sum_{\sigma} \sum_{i} \int d \mathbf{r} \varphi_{i}^{*}(\mathbf{x})\left(-\frac{1}{2} \nabla^{2}\right) \varphi_{i}(\mathbf{x}),
$$

поэтому

$$
E_{\mathrm{xc}}[\rho]=E_{\mathrm{xc}}^{\prime}[\rho]+T-T_{s}[\rho] .
$$

Разность $T-T_{s}[\rho]$ представляет собой так называемую корреляционную кинетическую энергию [5]. Последняя, согласно (4), должна давать вклад в $v_{\mathrm{xc}}(r)$, что и учитывает первый член в соотношении (42). Из сказанного выше ясно, что в случае системы взаимодействующих электронов подобный член появиться не может.

Конечно, в рамках приближений, использованных в работе [2], асимптотика первого члена в соотношении $(42)\left(\sim 1 / r^{5}\right)$ позволяет не обращать на него внимания. Но остается вопрос о знаке последних членов в выражениях (42) и (13). Здесь важно обратить внимание на второе из отмеченных следствий из смысла уравнения (11). По нашему мению, это следствие делает некорректной (см. (25)) простую подстановку функции $\varphi_{N}(\mathbf{x})$ вместо $f_{0}(\mathbf{x})$. Такой подход на самом деле озачает навязывание $\varphi_{N}(\mathbf{x})$ уравнения, которому эта функция подчиняться не обязана.

Согласно (44) функция $f_{s}(\mathbf{x})$ (при любых $s$ ) должна определяться соотношением

$$
f_{s}(\mathbf{x})=\phi(\mathbf{r}) g_{s}(\mathbf{x}),
$$

где

$$
g_{s}(\mathbf{x})=\langle N-1, s \mid \Phi(N-1)\rangle .
$$

Если справедливо уравнение (23), то, подставив в него соотношение (68), мы будем иметь

$$
\begin{aligned}
& g_{0}(\mathbf{x})(-\left.\frac{1}{2} \nabla^{2} \phi(\mathbf{r})\right)-\nabla g_{0}(\mathbf{x}) \nabla \phi(\mathbf{r})+\phi(\mathbf{r})\left(-\frac{1}{2} \nabla^{2} g_{0}(\mathbf{x})\right)+ \\
& \quad+\left[v(\mathbf{r})+\left\langle N-1,0\left|\sum_{j \geqslant 2} \frac{1}{\left|\mathbf{r}-\mathbf{r}_{j}\right|}\right| N-1,0\right\rangle-\frac{1}{r^{4}} \sum_{s>0} \frac{\left|D_{s 0}\right|^{2}}{\omega_{s}}\right] \phi(\mathbf{r}) g_{0}(\mathbf{x})= \\
&=\varepsilon_{0} \phi(\mathbf{r}) g_{0}(\mathbf{x}) .
\end{aligned}
$$

Функция $\phi(\mathbf{r})$ подчиняется точному уравнению $(40)$, которое при учете выражений $(15),(32),(38),(54)$ и в пренебрежении первым членом соотношения (42) приобретает вид

$$
\begin{gathered}
-\frac{1}{2} \nabla^{2} \phi(\mathbf{r})=\varepsilon_{0} \phi(\mathbf{r})-\left[v(\mathbf{r})+\left\langle N-1,0\left|\sum_{j \geqslant 2} \frac{1}{\left|\mathbf{r}-\mathbf{r}_{j}\right|}\right| N-1,0\right\rangle-\right. \\
\left.-\sum_{s>0} \omega_{s} \sum_{\sigma}|\langle N-1, s \mid \Phi(N-1)\rangle|^{2}\right] \phi(\mathbf{r}) .
\end{gathered}
$$


Согласно формулам (32), (47)

$$
\sum_{s>0} \omega_{s} \sum_{\sigma}|\langle N-1, s \mid \Phi(N-1)\rangle|^{2}=\frac{1}{r^{4}} \sum_{s>0} \frac{\left|D_{s 0}\right|^{2}}{\omega_{s}}
$$

Подставляя в уравнение (70) соотношение (71) и учитывая (72), получим

$$
-\nabla g_{0}(\mathbf{x}) \nabla \phi(\mathbf{r})-\phi(\mathbf{r}) \frac{1}{2} \nabla^{2} g_{0}(\mathbf{x})=2 \frac{1}{r^{4}} \sum_{s>0} \frac{\left|D_{s 0}\right|^{2}}{\omega_{s}}
$$

Согласно соотношениям (5), (16), в условиях (7), (41) можно сделать замену $\phi(\mathbf{r}) \rightarrow$ $\varphi_{N}(\mathbf{r})$. Поэтому, подставив выражение (73) в уравнение $(70)$, после деления на $g_{0}(\mathbf{x})$ получим

$$
\left(-\frac{1}{2} \nabla^{2}+v(\mathbf{r})+\left\langle N-1,0\left|\sum_{j \geqslant 2} \frac{1}{\left|\mathbf{r}-\mathbf{r}_{j}\right|}\right| N-1,0\right\rangle+\frac{1}{r^{4}} \sum_{s>0} \frac{\left|D_{s 0}\right|^{2}}{\omega_{s}}\right) \varphi_{N}(\mathbf{r})=\varepsilon_{0} \varphi_{N}(\mathbf{r}) .
$$

Таким образом, в рамках приближений работы [2] верны оба уравнения (23) и (74). Но с точки зрения смысла функций $f_{0}(\mathbf{x})$ и $\varphi_{N}(\mathbf{r})$ обменно-корреляционный потенциал должен, по нашему мнению, определяться выражением

$$
v_{\mathrm{xc}}(\mathbf{r})=\left\langle N-1,0\left|\sum_{j \geqslant 2} \frac{1}{\left|\mathbf{r}-\mathbf{r}_{j}\right|}\right| N-1,0\right\rangle-\int d \mathbf{r}^{\prime} \frac{\rho\left(\mathbf{r}^{\prime}\right)}{\left|\mathbf{r}-\mathbf{r}^{\prime}\right|}+\frac{1}{r^{4}} \sum_{s>0} \frac{\left|D_{s 0}\right|^{2}}{\omega_{s}} .
$$

\section{Список литературы}

[1] В. Кон. УФН. 2002. Т. 172. № 3. С. 336-348.

[2] C.-O. Almbladh, U. von Burth. Phys. Rev. B. 1985. V. 31. № 6. P. 3231-3244.

[3] M. Levy, J. P. Perdew, V. Sahni. Phys. Rev. A. 1984. V. 30. № 5. P. 2745-2748; G. Hunter. The exact Schrödinger equation for the electron density. In: Density Matrices and Density Functionals. Proc. of the A. John Coleman Symposium. Eds. R. Erdahl, V.H. Smith. Jr. Dordrecht: Reidel Publ., 1987. P. 583-596.

[4] Л.П. Гинзбург. ТМФ. 1999. Т. 121. № 3. С. 450-463.

[5] V. Sahni. Phys. Rev. A. 1997. V. 55. № 3. P. 1846-1856. 